\title{
Mycobacterium bovis BCG: the importance of an accurate identification in the diagnostic routine
}

\author{
Antonella Grottola, Anna Fabio, Anna MT Sabbatini, Giuliana Fabio, Mariagrazia Apice, Alessia Di Nauta, \\ Giulia Forbicini,Annunziata La Regina, Sara Tagliazucchi, Monica Pecorari, Fabio Rumpianesi, Chiara Casolari \\ Struttura Complessa di Microbiologia e Virologia, Azienda Integrata Ospedaliero-Universitaria, Modena
}

Key words: $M$. bovis BCG, bladder cancer, genotyping MTB complex

Mycobacterium bovis BCG: I'importanza di una precisa identificazione nella diagnostica routinaria

\section{SUMMARY}

M. bovis BCG is used clinically in the immunotherapy treatment of superficial bladder cancer to prevent progression to invasive disease, leading in some cases to a severe localized inflammation or disseminated infections. For this reason, an accurate and early identification of this particular microorganism is clinically relevant. We describe a case-report of bladder cancer with a urine culture-positive for mycobacteria initially diagnosed as MTB complex infection and later identified as BCG disease by molecular methods.

\section{INTRODUZIONE}

Il $M$. bovis BCG (Bacillus Calmette-Guérin) è un derivato attenuato di un ceppo virulento di Mycobacterium bovis, utilizzato sia come vaccino antitubercolare che come veicolo ricombinante per vaccini multivalenti nei riguardi di diversi agenti infettivi. Viene inoltre normalmente impiegato nel trattamento immunoterapico del carcinoma vescicale superficiale in base ad una sua comprovata efficacia nella profilassi delle recidive dopo resezione endoscopica e nella prevenzione della progressione alla malattia infiltrante (7). In presenza di severi deficit immunitari, a seguito di vaccinazioni o instillazioni endovescicali previste dai protocolli terapeutici, il BCG può determinare flogosi localizzate o gravi infezioni disseminate (4). Per questo motivo appare oggi clinicamente rilevante una precisa e rapida identificazione di questo particolare microorganismo.

I ceppi BCG attualmente utilizzati nel mondo, strettamente correlati ai microorganismi appartenenti al Mycobacterium tuberculosis complex, (MTB complex) sono fenotipicamente e geneticamente simili ai ceppi di $M$. bovis, diversificandosi da questi per l' attenuazione di virulenza ottenuta con continui passaggi in vitro. Le caratteristiche biochimiche e colturali non forniscono dati identificativi definitivi così come gli stessi metodi molecolari più diffusamente impiegati nella diagnostica per la ricerca di MTB complex non sono in grado di differenziarne le singole specie all'interno del raggruppamento, fornendo risultati genericamente positivi. L'analisi comparativa del genoma di $M$. tuberculosis e BCG ha mostrato che 16 regioni geniche (RDs) del M. tuberculosis sono delete o mancanti in alcuni o in tutti i ceppi di M. bovis e/o BCG. Tra queste, 11 regioni del M. tuberculosis H37Rv (RD1, da RD4 a RD7, da RD9 a RD13, RD15) sono assenti in tutti i ceppi di BCG $(1,2)$. In particolare la regione genomica RD1 viene utilizzata come marker per l'individuazione specifica di BCG $(11,8)$.

\section{Descrizione del caso}

Nel settembre 2006 pervenivano al nostro laboratorio 3 campioni di urina di 3 giorni consecutivi da un paziente di 77 anni ricoverato presso il reparto di Urologia del Policlinico di Modena, per ricerca di micobatteri. L'esame batterioscopico diretto per ricerca di bacilli alcool-acido resistenti risultava negativo per tutti e tre i campioni, sia dopo colorazione di Ziehl Neelsen che di Truant. Venivano allestiti gli esami colturali su terreni liquidi (Bactec MIGIT 960, Becton Dickinson, Milano, Italia) e solidi (Lowenstein Jensen, bioMérieux, Milano, Italia).

La coltura in terreno liquido di uno dei tre campioni si positivizzava nell'arco di 18 giorni. Alcuni giorni dopo si rende- va evidente la crescita anche su terreno solido. Le colonie, non pigmentate e rugose, presentavano l'aspetto tipico proprio dei bacilli tubercolari. L'esame microscopico a partire dalla coltura mostrava bacilli alcool-acido resistenti di forma allungata. Il riconoscimento del ceppo con i metodi molecolari in uso (Amplified MTD, bioMérieux, Milano, Italia) indicava MTB complex. L'antibiogramma effettuato in terreno liquido (MIGIT) secondo la metodica standard denotava la sensibilità del ceppo a Etambutolo (5 e $7.50 \mathrm{mcg} / \mathrm{ml}$ ), Rifampicina (1 mcg/ml), Streptomicina (1 e $4 \mathrm{mcg} / \mathrm{ml})$, mentre nei riguardi di isoniazide si rilevava sensibilità a $0.40 \mathrm{mcg} / \mathrm{ml}$ e resistenza alla concentrazione più bassa di $0.10 \mathrm{mcg} / \mathrm{ml}$. L'informazione veniva trasmessa tempestivamente al clinico che tuttavia valutava come incongruo il dato e ricusava l'ipotesi di infezione tubercolare informando il laboratorio di un verosimile errore nella richiesta. Il paziente era portatore di un carcinoma uroteliale vescicale plurifocale asportato 3 mesi prima e stava praticando settimanalmente instillazioni endovescicali con BCG. Il ceppo isolato veniva allora sottoposto ad ulteriori e più complete prove molecolari e identificato come BCG.

A tale scopo, per l'identificazione veniva utilizzato il kit Genotype MTBC (Arnika s.r.l., Milano, Italia); questo test genotipico molecolare, basato sulla tecnologia DNA-Strip, permette di differenziare BCG da altre specie appartenenti a MTB complex in base ai polimorfismi del gene gyrase B e delezione di RD1 (10). Per maggior sicurezza e per escludere definitivamente un'infezione tubercolare, venivano ripetute indagini colturali per micobatteri in successivi campioni di urina, con esito negativo. Il test QuantiFERON-TB Gold (Cellestis, Ltd, Carnegie, Victoria, Australia) effettuato su plasma risultava negativo e pertanto veniva esclusa un'infezione tubercolare latente.

$\mathrm{Al}$ fine di indagare un eventuale processo flogistico da BCG in atto, ulteriori test molecolari venivano effettuati su campioni bioptici vescicali con risultati negativi. Nel dicembre 2006 le indagini istologiche confermavano la presenza del carcinoma uroteliale e veniva impostata una terapia con mitomicina. Nel giugno 2007 veniva effettuata una cistoscopia con biopsia che dava esito negativo.

\section{Commento}

L'isolamento colturale da materiale biologico rappresenta il "gold standard" per la diagnosi di tubercolosi attiva; tuttavia la necessità di trattare il paziente tempestivamente e di limitare la diffusione di ceppi di micobatteri farmacoresistenti (Multidrug-Resistant TB e XDR-TB) ha favorito l'utilizzo di nuove tecniche di biologia molecolare molto più sensibili e veloci accanto alle metodiche microbiologiche tradizionali.

\section{Corresponding author: Antonella Grottola}

SC Microbiologia e Virologia, Dip. Attività Integrate di Laboratori, Anatomia Patologica e Medicina Legale, AOU, Policlinico di Modena Via del Pozzo7I, 4I I00 Modena, ITALY - Tel. +39 0594223752 - Fax. +39 0594223625

E-mail: antonella.grottola@unimore.it 
L’identificazione di tutti i ceppi di micobatteri isolati ricopre un ruolo chiave nella pratica clinica al fine di avere una diagnosi definitiva e conseguentemente per impostare una corretta terapia in tempi rapidi. Tuttavia per MTB complex può essere accettata un'identificazione generica a livello di raggruppamento senza arrivare all'identificazione di specie. La nostra esperienza, basata sull'isolamento di micobatteri in un paziente con carcinoma vescicale, inizialmente diagnosticato come MTB complex e solo successivamente identificato come BCG, focalizza l'attenzione sulla nececessità di non fermarsi ad una identificazione generica ma di procedere all'identificazione nell'ambito dell'MTB complex compatibilmente con il quadro clinico del paziente.

Il BCG presente nell'urina cresce in coltura e a tutti gli effetti può essere identificato in prima battuta come M. tuberculosis. È pertanto assolutamente indispensabile in questi casi richiedere e valutare accuratamente i dati clinico-anamnestici del paziente e procedere ad ulteriori prove per identificare esattamente l'isolato. La sensibilità all'idrazide dell'acido tiofene-carbossilico (TCH) discrimina $M$. bovis da $M$. tuberculosis ma non differenzia i differenti ceppi BCG impiegati nei diversi trattamenti preventivi e terapeutici. Vista l'impossibilità di riconoscere i ceppi attenuati di BCG dagli altri ceppi del MTB complex in base all'osservazione dei caratteri fenotipici, i metodi molecolari hanno dimostrato di essere strumenti utili per una rapida differenziazione di specie $(9$, 5); infatti il kit Genotype MTBC da noi utilizzato in questo caso, ha consentito una identificazione del ceppo in esame come appartenente alla specie BCG.

Il BCG sta assumendo oggi un interesse emergente come responsabile di quadri clinici di diversa gravità; a seguito di instillazioni endovescicali in pazienti portatori di carcinoma uroepiteliale, oltre a processi localizzati di flogosi cronica, vengono segnalati in letteratura casi di orchiepididimite, mentre casi post-vaccinali di infezione disseminata e di tubercolosi cutanea sono descritti in pazienti immunodepressi (6). Appare quindi indispensabile prendere in considerazione questa problematica ed essere consapevoli dell'importanza di una identificazione di specie all'interno del MTB complex applicando una diagnostica microbiologica adeguata nella routine della pratica di laboratorio e clinica. Questo vale soprattutto in presenza di pazienti con immunodeficienze primarie, per i quali i CDC suggeriscono precisi criteri diagnostici clinici e di laboratorio per la diagnosi di infezione da BCG (3). Una diagnosi differenziale in tal senso dovrebbe sempre essere posta in presenza di grave e persistente stato infiammatorio con formazione di granulomi. L'identificazione di MTB complex in biologia molecolare da campioni bioptici, pur senza differenziazione di BCG, in pre- senza di lesioni infiammatorie granulomatose può essere significativa di infezione probabile, mentre la diagnosi definitiva comporta l'identificazione di BCG dai campioni d'organo tramite coltura o metodi molecolari, in presenza di tipiche alterazioni istopatologiche. Sintomi di coinvolgimento sistemico come febbre e calo ponderale sono elementi importanti da associare al dato di laboratorio.

La presente segnalazione di un caso di positività colturale urinaria per micobatteri in un paziente con carcinoma vescicale, inizialmente identificato come MTB complex e solo successivamente identificato come BCG con ulteriori test molecolari, contribuisce a sottolineare l'importanza di effettuare sempre una precisa identificazione di specie, infatti una generica positività per MTB complex può fuorviare la diagnosi e comportare l'avvio di un trattamento terapeutico inadeguato.

In conclusione, sulla base della esperienza di questo "casereport”, possiamo ancora una volta sottolineare come sia importante ed indispensabile una stretta collaborazione tra clinici e microbiologici per arrivare ad una corretta e rapida diagnosi e quindi alla risoluzione completa del caso clinico.

\section{BIBLIOGRAFIA}

1. Al-Attiyah R, Mustafa AS. Characterization of human cellular immune responses to novel Mycobacterium tuberculosis antigens encoded by genomic regions absent in Mycobacterium bovis BCG. Infect Immun 2008; 76: 4190-8.

2. Behr MA, Wilson MA, Gill WP, et al. Comparative genomics of BCG vaccines by whole-genome DNA microarray. Science 1999; 8: 1520-3.

3. Bernatowska EA, Wolska-Kusnierz B, Pac M, et al. Disseminated Bacillus Calmette-Guérin Infection and Immunodeficiency. Emerg Infect Dis 2007; 13 (5): 799-801 http://www.cdc.gov/eid/content/13/5/799.htm

4. Borrè S, Brustia D, Rosa F, Brondolo R, Rizzo G, Garavelli PL. CalmetteGuérin bacillus disseminated infection after intravesical instillation. Recenti Prog Med 2002; 93: 247-8.

5. Djelouadji Z, Raoult D, Daffè M, Drancourt M. A single-step sequencing method for the identification of Mycobacterium tuberculosis complex species. PLoS Negl Trop Dis. 2008; 2 (6): 253.

6. Harada H, Seki M, Shinojima H, Masayoshi M, Hirano T, Togashi M. Epididymo-orchitis caused by intravesically instillated bacillus CalmetteGuérin: genetically proven using multiplex polymerase chain reaction method. Int J Urol 2006; 13: 183-5.

7. Morales A, Eidinger D, Bruce AW. Intracavitary bacillus Calmette-Guérin in the treatment of superficial bladder tumors. J Urol 1976; 116: 180-3.

8. Okazaki T, Ebihara S, Takahashi H, et al. Multiplex PCR-identified cutaneous tuberculosis evoked by Mycobacterium bovis BCG vaccination in Healthy baby. J Clin Microbiol 2005; 43: 523-5.

9. Ricther E, Weizenegger M, Fahr A-M, Rüsch-Gerdes S. Usefulness of genotype MTBc assay for differentiating species of the Mycobacterium tuberculosis complex in cultures obtained from clinical specimens. J Clin Microbiol 2004; 42: 4303-6.

10. Ricther E, Weizenegger M, Rüsch-Gerdes S, Niemann S. Evaluation of Genotype MTBC Assay for differentiation of Clinical Mycobacterium tuberculosis complex isolates. J Clin Microbiol 2003; 41: 2672-5.

11. Talbot EA, Williams DL, Frothingham R. PCR identification of Mycobacterium bovis BCG. J Clin Microbiol 1997; 35: 566-9. 